\title{
Gaussian -Diophantine quadruples with property D (1)
}

\author{
${ }^{1}$ S.Vidhyalakshmi $*,{ }^{2}$ M. A. Gopalan and ${ }^{3}$ K. Lakshmi \\ ${ }^{1,2,3}$ Department of Mathematics, Shrimati Indira Gandhi College,Trichy-620002.
}

\begin{abstract}
A set of $m$ Gaussian integers is called a complex Diophantine m-tuple with the property $D(z)$ if the product of its any two distinct elements increased by $z$ is a square of a Gaussian integer. In this paper, we present Gaussian-Diophantine quadruples with property D(1).Few examples of complex Diophantine quadruples with the property $D(1)$ are presented.
\end{abstract}

Keywords: Diophantine quadruples, Integral solutions, Gaussian integers, Pell equation

\section{Introduction}

A set of positive integers $\left\{a_{1}, a_{2}, a_{3} \ldots \ldots . a_{m}\right\}$ is said to have the property $\mathrm{D}(\mathrm{n}), n \in Z-\{0\}$,

if $a_{i} a_{j}+n$ is a perfect square for all $1 \leq i \leq j \leq m$ and such a set is called a Diophantine m-tuples with property $\mathrm{D}(\mathrm{n})$. Many mathematicians considered the problem of the existence of a Diophantine quadruples with property $\mathrm{D}(\mathrm{n})$ for any arbitrary integer $\mathrm{n}[1]$ and also, for any linear polynomials in $\mathrm{n}$. Further various authors considered the connections of the problem of Diaphanous, Davenport and Fibonacci numbers in [2-21].

In this paper we consider the analogous problem for Gaussian integers. Let $\mathrm{z}$ be a Gaussian integer and let $m \geq 2$ be an integer. A set $\left\{a_{1}, a_{2}, a_{3} \ldots \ldots a_{m}\right\} \subset Z(i) \backslash\{0\}$ is said to have this property $\mathrm{D}(\mathrm{z})$ if the product of its any two distinct elements increased by $\mathrm{z}$ is a square of a Gaussian integer. If the set $\left\{a_{1}, a_{2}, a_{3} \ldots \ldots . . a_{m}\right\}$ is a complex Diophantine quadruple then the same is true for the set $\left\{-a_{1},-a_{2},-a_{3} \ldots \ldots . . .-a_{m}\right\}$.Particularly in [22], the authors have analyzed the problem of the existence of the complex Diophantine quadruples. In this paper, we present a Gaussian -Diophantine quadruple with property $\mathrm{D}(1)$.

\section{MSC 2000 Mathematics subject classification: 11D99}

\section{Method of analysis:}

To start with, it is seen that the pair $(a, b)$ is Gaussian Diophantine 2-tuples with property D (1)

where $a$ and $b$ are Gaussian integers of the form

$$
\begin{aligned}
& a=\left(2 p^{2}-2 q^{2}-p\right)+i(4 p q-q) \text { and } \\
& b=\left(2 p^{2}-2 q^{2}+7 p+6\right)+i(4 p q+7 q)
\end{aligned}
$$

Let $c_{s}$ be any non zero integer such that

$$
\begin{aligned}
& \mathrm{a} * c_{s}+1=\alpha_{s}^{2} \\
& \mathrm{~b} * c_{s}+1=\beta_{s}^{2}
\end{aligned}
$$

Eliminating $c_{S}$ between (1) and (2) we get

$$
b \alpha_{s}^{2}-\mathrm{a} \beta_{s}^{2}=b-a
$$

Substitution of the linear transformations

$$
\begin{aligned}
& \alpha_{s}=X_{s}+a T_{s} \\
& \beta_{s}=X_{s}+b T_{s}
\end{aligned}
$$

\footnotetext{
* The financial support from the UGC, New Delhi (F.MRP-5123/14 (SERO/UGC) dated March 2014) for a part of this work is gratefully acknowledged
} 
in (3) leads to the equation

$$
X_{s}^{2}=a b T_{s}^{2}+1
$$

where

$$
\begin{aligned}
a b=\left(4 p^{4}+4 q^{4}-24 p^{2} p^{2}-36 p q^{2}+12 q^{3}+5 p^{2}-5 q^{2}-6 p\right)+ \\
i\left(16 p^{3} q-16 p q^{3}+36 p^{2} q-12 q^{3}+10 p q-6 q\right)
\end{aligned}
$$

The general solution of (6) is given by

$$
\left.\begin{array}{l}
X_{s}=\frac{1}{2}\left[\left(X_{0}+\sqrt{a b} T_{0}\right)^{s+1}+\left(X_{0}-\sqrt{a b} T_{0}\right)^{s+1}\right] \\
T_{s}=\frac{1}{2 \sqrt{a b}}\left[\left(X_{0}+\sqrt{a b} T_{0}\right)^{s+1}-\left(X_{0}-\sqrt{a b} T_{0}\right)^{s+1}\right]
\end{array}\right\}
$$

Taking $s=0$ in (7) and using (4) we have

$$
\alpha_{0}=\left(4 p^{2}-4 q^{2}+2 p-1\right)+i(8 p q-2 q)
$$

In view of (1) we have

$$
c_{0}=\left(8 p^{2}-8 q^{2}+12 p+4\right)+i(16 p q+12 q)
$$

Observe that

$$
\begin{aligned}
\left\{\left(2 p^{2}-2 q^{2}-p\right)+i(4 p q-q),\right. & \left(2 p^{2}-2 q^{2}+7 p+6\right)+i(4 p q+7 q), \\
& \left.\left(8 p^{2}-8 q^{2}+12 p+4\right)+i(16 p q+12 q)\right\}
\end{aligned}
$$

is a Gaussian Diophantine triple with property $\mathrm{D}(1)$

Again taking $s=1$ in (7) and using (4) we obtain

$$
\left.\begin{array}{rl}
\alpha_{1}=\left(16 p^{4}+16 q^{4}-88 p^{2} q^{2}+\right. & \left.32 p^{3}-76 p q^{2}-10 p-20 p q^{2}+1\right)+ \\
& i\left(64 p^{3} q-64 p q^{3}+96 p^{2} q-32 q^{3}-10 q\right)
\end{array}\right\}
$$

In view of (1) we have

$$
\begin{aligned}
& c_{1}=\left\{128\left(p^{6}-15 p^{4} q^{2}+15 p^{2} q^{4}-q^{6}\right)+576\left(p^{5}-10 p^{3} q^{2}+5 p q^{4}\right)+\right. \\
&\left.800\left(p^{4}-6 p^{2} q^{2}+q^{4}\right)+240\left(p^{3}-3 p q^{2}\right)-184\left(p^{2}-q^{2}\right)-60 p+20\right\} \\
&+i\left\{128\left(6 p^{5} q-20 p^{3} q^{3}+6 q^{5} p\right)+576\left(5 p^{4} q-10 p^{2} q^{3}+q^{5}\right)+\right. \\
&\left.800\left(4 p^{3} q-4 p q^{3}\right)+240\left(3 p^{2} q-q^{3}\right)-368 p q-60 q\right\}
\end{aligned}
$$

Hence

$$
\begin{gathered}
\left\{\left(2 p^{2}-2 q^{2}-p\right)+i(4 p q-q),\left(2 p^{2}-2 q^{2}+7 p+6\right)+i(4 p q+7 q),\right. \\
\left(8 p^{2}-8 q^{2}+12 p+4\right)+i(16 p q+12 q), \\
\left(128\left(p^{6}-15 p^{4} q^{2}+15 p^{2} q^{4}-q^{6}\right)+576\left(p^{5}-10 p^{3} q^{2}+5 p q^{4}\right)+\right. \\
\left.800\left(p^{4}-6 p^{2} q^{2}+q^{4}\right)+240\left(p^{3}-3 p q^{2}\right)-184\left(p^{2}-q^{2}\right)-60 p+20\right) \\
+i\left(128\left(6 p^{5} q-20 p^{3} q^{3}+6 q^{5} p\right)+576\left(5 p^{4} q-10 p^{2} q^{3}+q^{5}\right)+\right. \\
\left.\left.800\left(4 p^{3} q-4 p q^{3}\right)+240\left(3 p^{2} q-q^{3}\right)-368 p q-60 q\right)\right\}
\end{gathered}
$$

is a Gaussian Diophantine quadruple with property $\mathrm{D}(1)$

The repetition of the above process leads to the generation of infinitely many Gaussian Diophantine quadruples with property $\mathrm{D}(1)$ 
Table: Examples

\begin{tabular}{|l|l|}
\hline$(p, q)$ & Diophantine quadruple with property $\mathrm{D}(1)$ \\
\hline$(0,1)$ & $\{-2-i, 4+7 i,-4+12 i, 876+276 i\}$ \\
\hline$(1,1)$ & $\{-1+3 i, 13+11 i, 16+28 i,-6024-3276 i\}$ \\
\hline$(1,2)$ & $\{-7+6 i, 7+22 i,-8+56 i, 30864-36792 i\}$ \\
\hline
\end{tabular}

Note:

If $\left\{z_{1}, z_{2}, z_{3}, z_{4}\right\}$ is a quadruple with the property $D(z)$, then $\left\{\overline{z_{1}}, \overline{z_{2}}, \overline{z_{3}}, \overline{z_{4}}\right\}$ is a quadruple with the property, $D(\bar{z}), z_{1}, z_{2}, z_{3}, z_{4} \in Z(i)$

\section{Conclusion}

In this paper, we have presented a Gaussian Diophantine quadruple with property $\mathrm{D}(1)$. One may search for Gaussian Diophantine quadruples consisting of special numbers with suitable properties.

\section{References}

[1]. Balker.A, Duvemport.H; "The equations $3 x^{2}-2=y^{2}$ and $8 x^{2}-7=z^{2}$,.. Quart.J.Math.Oxford Ser, 1969,20 (2),129-137.

[2]. Hoggatt.V.E,Bergum.G.E,"A Problem of Fermat and the Fibonacci Sequence",Fibonacci Quart,1977;15:323-330.

[3]. Horadam.A.F,"Generalization of a result of Morgudo",Portugaliae Math,1987;44:131-136

[4]. Jones.B.E,"Asecond variation on a problem of DiophPantus and Devenport",Fibonacci Quart,1978; 16:155-165

[5]. Long.C,Bergum.G.E,"On a problem of Diophantus,in:Application of Fibonacci Numbers.(Philippou.A.N, Horadam.A.F, Bergum.G.E,Eds.),Kluwer,Dordrecht,vol.2,1998,183-191

[6]. Gupta H, Singh K, "On k-triad sequences", Internat.J. Math. Math. Sci. 1985;5:799-804.

[7]. Morgado.J," Generalization of a result of Hoggatt.V.E,Bergum on Fibonacci numbers", Portugaliae Math,1983-1984,42:441-445

[8]. Morgado.J,"Note on a Shannon's theorem concerning the Fibonacci numbers ", Portugaliae Math, 1991;48:429-439

[9]. Morgado.J,"Note on the Chebyshev polynomials and applications to the Fibonacci numbers", Portugaliae Math, 1995;52:363-378

[10]. Udrea.G.H,"A Problem of Diophantos-Fermat and Chebychev Polynomials of the second kind" Portugaliae Math, 1995;52:301-304

[11]. Brown E; "Sets in which $x y+k$ is always a square", Math.Comp, 1985; 45:613-620.

[12]. Beardon A.F, Deshpande M.N; "Diophantine triples". The Mathematical Gazette, 2002; 86:258-260.

[13]. Deshpande M.N," Families of Diophantine triplets", Bulletin of the Marathwada Mathematical Society, 2003; 4, 19-21.

[14]. Bugeaud .Y,Dujella.A and Mignotte.M," On the family of Diophantine triples $\left(k-1, k+1,16 k^{3}-4 k\right)$ ", Glasgow Math.j,2007,49:333-344

[15]. Fujita Y, "The extensibility of Diophantine pairs $(k-1, k+1)$ ”, J. Number Theory, 2008;128:322-353

[16]. Deshpande M.N,” One interesting family of Diophantine triples”. Internet J. Math.ed. Sci. Tech,2012;33:253-256.

[17]. Gopalan M.A, Pandichelvi V," On the Extendability of the Diophantine triple involving Jacobsthal numbers $\left(J_{2 n-1}, J_{2 n+1}-3,2 J_{2 n}+J_{2 n-1}+J_{2 n+1}-3\right) \quad$ "International Journal of Mathematics \& Applications,2(1), June $\quad 2009,1-3$

[18]. Srivihya G,” Diophantine Quadruples for Fibboncci numbers with property D(1)" Indian Journal of Mathematics and Mathematical Science, 2009; 5(2):57-59.

[19]. Gopalan M.A, Srividhya G,’Two special Diophantine triples”. Diophantus J.Math, 2012;1(1): 23-27

[20]. Gopalan M.A, Srividhya G; "Diophantine Quadraple for Fibonacci and Lucas numbers with property D(4) “ Diophantus J.Math. 2012; 1(1):15-18.

[21]. Gopalan MA, Srividhya G; Two special Diophantine triples. Diophantus J.Math, 2012; 1(1): 23-27

[22]. Andrej Dujella,Zagreb,Croatia,’The Problem of Diophantus and Davenport for Gaussian Integers” Glas.Mat.Ser.III 32(1997), 1-10 